\title{
INJURIES TO THE EYE WITH REPORT OF 1051 CASES.
}

\author{
Don M. Campbell, M.D., L.R.C.S., (Edinb.) \\ John M. Carter, A.M., M.D. \\ DETROIT, MICH.
}

This is an analytic account of the cases scen in a period of two years, giving the kind of history obtained, the clinical data brought out in the two classes of cases, minor injuries and major. The diagnosis including the importance of the history and the use of the X-rays is discussed. The removal of foreign bodies from inside of the eye is considered with results from twenty-four magnet extractions. The importance of early diagnosis and treatment is insisted upon.

It is common knowledge that the medical profession, in general, finds it difficult to be interested in any paper or discussion concerning the eye. This lack of interest manifests itself early, in fact, at the time we receive our first instruction in ophthalmology as medical students. One of the great reasons for this lack of interest is the absence of evident practical application in the subject matter presented.

Injuries to the eye, their proper treatment and management, will always be classed as one of the most important subjects that the oculist has in his work. In many ways it is none the less important to the medical profession in general. The object of this paper is to give a synopsis of 1051 cases of industrial injuries to the eye, which have been treated in our office in the past two years. We endeavor to emphasize those points which, to us, seem most important and of practical interest to the oculist and to the general medical profession.

In treatment of the eye, as in other branches of medicine, a good history and a carefully kept record should be the first essentials. We have taken for this series, as nearly as possible, a uniform history covering the following points :

1. When injured.

2. The type of work engaged in when injured.

3. The nature of the injury.

4. A statement concerning previous disease of the eye or eye injury; also concerning such subjective symptoms as pain, photophobia or blurring of the sight.

The vision in each eye was recorded at the time of the first visit at the of- fice, also at later visits, and upon discharge; careful record was made of the condition found at the initial examination and of the subsequent progress of the case. We have mailed to the company employing the man injured, a copy of the original record, also a copy of the notes made at the subsequent visits and of all $\mathrm{X}$-ray and laboratory reports. When the case is closed the company has an exact duplicate of the record as it occurs in our office.

Such a scheme has many advantages, some of the most noteworthy being that it enables "the shop" to be in constant touch with the progress of the case, also to "follow up" and see that their men come regularly for treatment. It at once stimulates and maintains an interest in the case.

$$
\text { CLINICAL DATA. }
$$

From a study of such a series one is able to determine a number of essentially important factors in eye injury. The data accumulated will show:

1. The type of work responsible for the greatest number of our cases.

2. The work offering the greatest number of serious injuries and the converse.

3. What per cent are seen at an early date following the injury, and an approximate estimate of the influence this has on the recovery of the case.

4. We will sec emphasized the importance of the $X-r a y$ in the diagnosis of metallic bodies, and the valuable assistance it affords the surgeon who is called upon to remove same.

5. We can determine what per cent of eyes have been lost and some of the contributing factors. These are the practical points we wish to emphasize.

In our analysis of this series we find the type of work responsible for the 


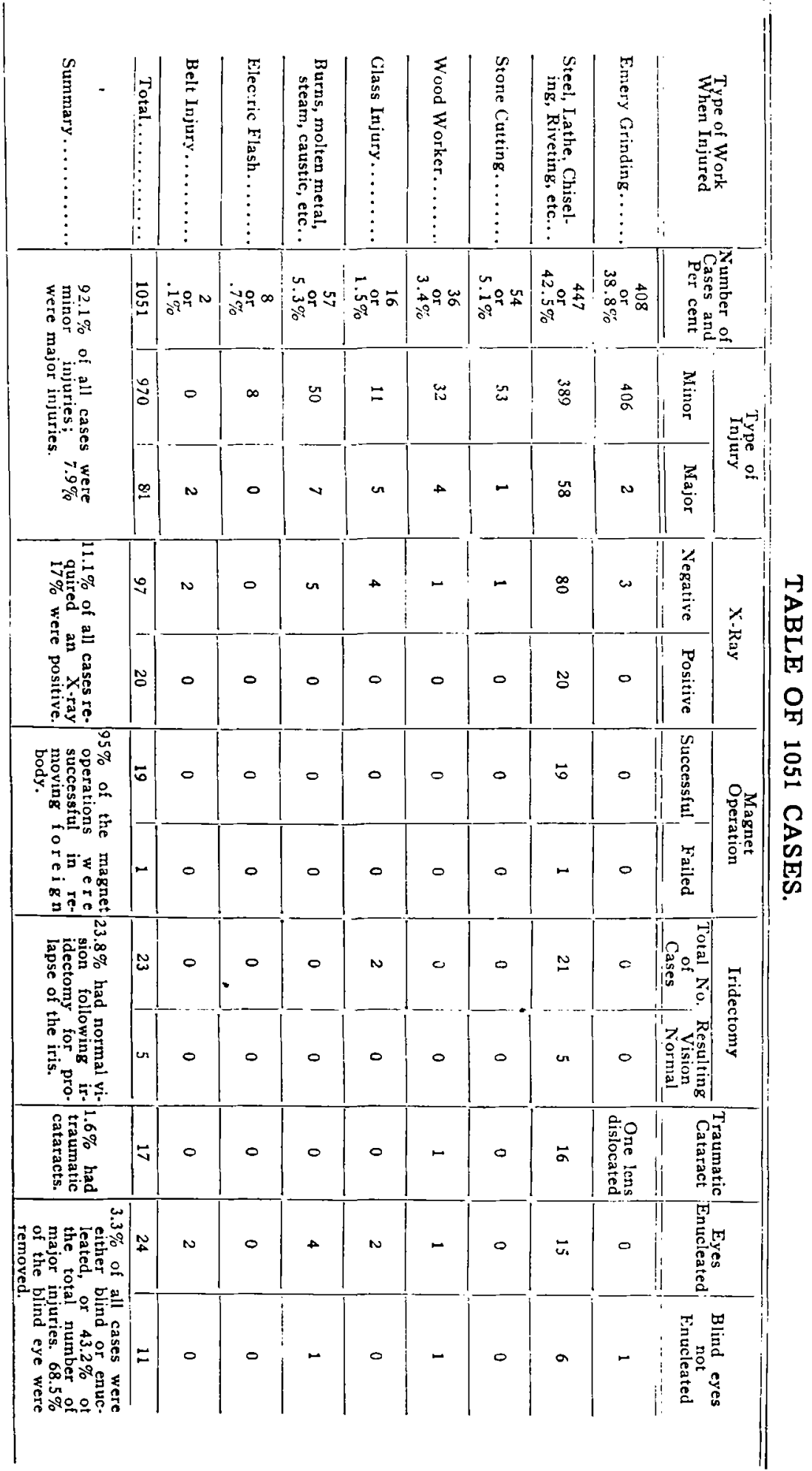


greatest number of our cases is seen to be the steel group, with emery grinding as a close second; the latter having just 37 cases less than the former, and the two groups together being responsible for $81.3 \%$ of all our cases. We see, therefore, that in eye injuries of this series we have patients coming from two great classes of work-those working on steel, like hammering, riveting, drilling, etc., and those working on emery.

For the purpose of study we may divide these cases into two groups: 1. The minor injuries; those with foreign bodies embedded in the coats of the eyeball and not perforating to the interior of the eye; nonperforating cuts and lacerations: first and second degree burns, etc. Most of the cases come in this group. 2. The major injuries; those in which the injury is of a more serious nature; such as perforating wounds of the eyeball with and without retained foreign bodies, third degree burns, and rupture or detachment of the coats of the eye. Of the total 1051 cases, 81 are found to come in the group of more serious or major injuries, or, in other words, $7.9 \%$ of the cases sent to our office have proven to be major injuries. This percentage is considerably lower when figured on the total number of injuries occurring in a large plant where all the cases are cared for.

The greatest number of our minor injuries occurred in the emery class, 406 cases, as compared with the next largest number, 389 , in the steel group. In reality, as to the occurrence of minor injuries, there is little difference in these two classes of work, only 17 cases more occurring in the emery than in the steel. Quite the converse is true when we look at the major injuries. Fifty-eight, or $71 \%$ of the total number of the major injuries, are chargeable to steel work, and only two major injuries occurred in the emery class. To know the type of work the patient was engaged in when injured is of no little importance. It should be noted on the history in the form of a permanent record.

We have been interested to note whether one eye is more often injured than the other. In 1906 Sweet $^{1}$ reported a series of 420 cases of eye injuries from foreign bodies. In these he found that
178 were injured in the right eye and 242 were injured in the left eye-54 more in the left in a series of 420 . He explains the prevalence of injuries to the left eye as being due to the right hand position, which the majority of men assume while working. In our series the two eyes share more equally, only 23 more being injured in the left than in the right, in a series of 1051 cases. We would conclude that the two eyes share about equally.

The small number of major injuries, only $7.9 \%$, would seem to indicate that much has been accomplished by the "Safety First" campaigns and by the efficiency engineer. We believe that many of what originally are simple minor injuries, thru one cause or another, become or are converted into a more serious condition. It is this feature with which we are particularly concerned in this paper. It is here that the general medical profession can be of great assistance. The delay in proper treatment is one of the most potent factors responsible for permanent injury to the eye. It is here that we should seek closer cooperation between the physician, who sees the case in general practice, and the oculist.

In trying to make some estimate of the time which intervenes between the occurrence of injury and treatment, we find in this series that only $17.5 \%$ were seen at our office on the day that they were injured, $43.5 \%$ on the second day, $17.5 \%$ on the third day, and $21.5 \%$ waited four days or longer before they applied at our office for treatment. We consider these figures very significant. When we know that routine culture shows that pathogenic organisms can be grown from a large number of the apparently normal conjunctivas, the importance of prompt attention to the injured eye becomes apparent.

The outer layers of the eye, and especially of the cornea, act as nature's barrier to infection, just as an intact skin surface does not permit organisms to pass and infect the deeper tissues. When the outer layers of the cornea are destroyed, either by the instrument used to remove the foreign body or by the foreign body itself, nature's means 
of preventing infection has been greatly handicapped. A most unpleasant chain of events may follow; the case may go on to ulceration and abscess of the cornea, or hypopion and perforation, then infection of the vitreous and panophthalmitis, a condition requiring that the eye be enucleated, while in the case limited to abscess of the cornea, healing may leave a dense scar which will permanently impair the sight. carliest possible moment, and in all cases where the iris has prolapsed between the cut surfaces of the wound. If this condition is neglected and the wound is left gaping, infection may extend to the interior of the eye. If this catastrophe is escaped the iris will become firmly healed into the corneal scar. This point of anchorage will be a constant source of irritation to the injured eye, often resulting in iridocy-

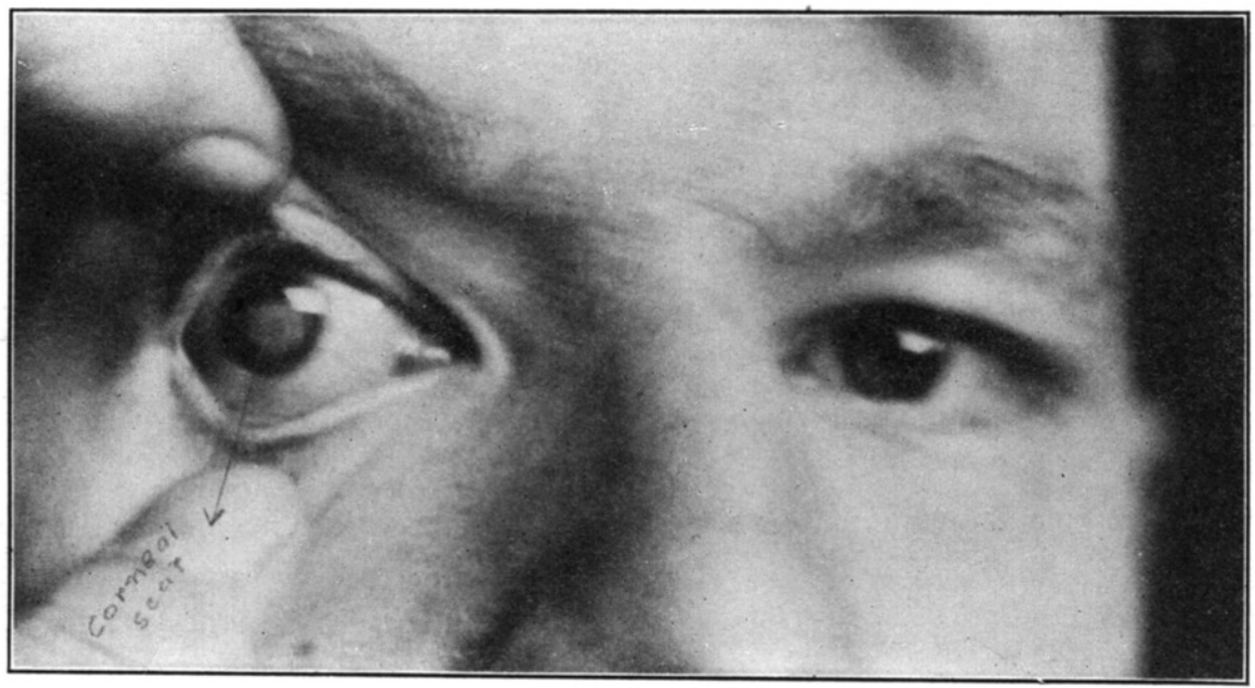

Fig. 1.-Extensive scarring of the right cornea resulting in permanent inpairment of the sight. The result of infection following a minor injury; a foreign body embedded in outer layers of cornea. Was not seen until four days following injury, when he showed extensive abscess.

Thus we see that a simple minor injury of a foreign body embedded in the cornea, that is not promptly and properly treated, may cause permanent impairment or even complete loss of an eye.

The time element is even more important in the case of the major injuries. In this group we find two types of cases which are most often pitfalls for the medical profession. First, the cases with a perforating wound and a prolapse of the iris. As a rule, the perforation will be thru the cornea and the iris is found sticking out between the margins of the wound; it is here that a simple operative procedure, one not requiring even a general anesthetic, when promptly done, will often save an eye. The operation we speak of is an iridectomy. It should be done at the clitis and sccondary glaucoma, and may even cause a sympathetic involvement of the uninjured eye. Also, the pull exerted by the adhesion will distort the contour of the cornea and cause the vision to be greatly reduced. If the lens has not been injured, and the case is seen carly, a very satisfactory result may follow an iridectomy. In this series 23 cases required an iridectomy, 6 , or $23.8 \%$, having a normal vision after the operation. Without an operation the vision in most of these would certainly have been very poor, and many of the eyes would possibly have had to be enucleated.

The second group of cases in which we are most likely to make a mistake is where we fail to recognize that a foreign body is retained within the eye. It is in this group of cases that the 
$\mathrm{X}$-ray is our most valuable aid, not only in making the proper diagnosis but also in determining what shall be our plan of procedure. First, we must impress upon ourselves the extreme importance of the removal of all foreign bodies retained within the eye, at the carliest possible moment. This means that the diagnosis must be made not at the second, third or fourth visit the man makes at the office, but the diagnosis eled if one would diagnose the case early. The importance of the history is emphasized by the fact that twenty of our X-rays were positive for metal inside the eye, and all of these occurred in the steel group. We should go on the assumption that all cases with a history of injury while working with metal have a foreign body retained inside the eye, and then set about to prove or to disprove it at the first visit.

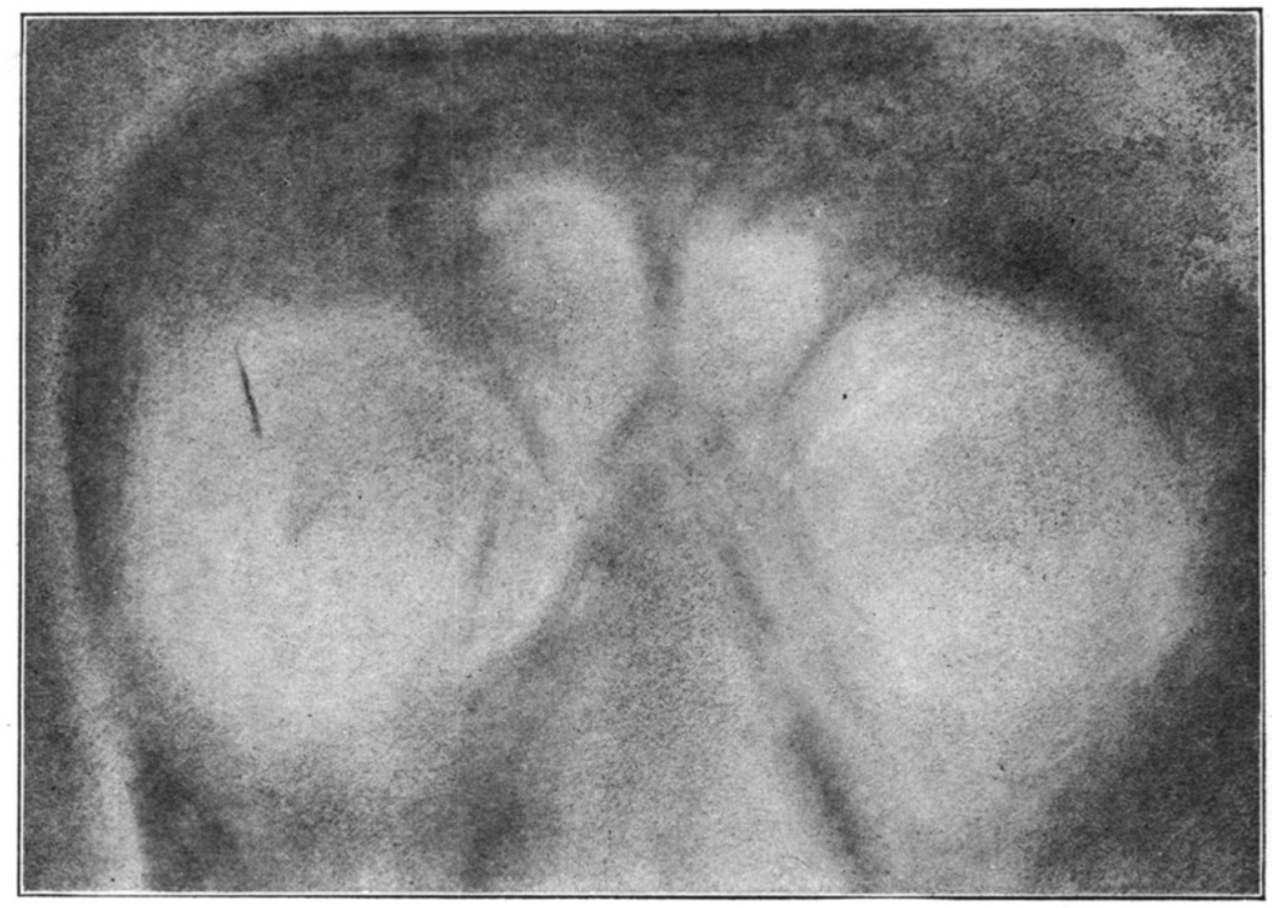

Fig. 2.- X-ray taken in the early part of 1897 of a long sliver of steel in the right eye, which was successfully removed by a magnet. Shown here for historic interest.

must be made on the first visit, and if the steel is within the eye it must be removed as soon as possible, the one exception being where the foreign body is completely embedded in the lens.

DIAGNOSIS.

Onc may ask: How can we most often make the diagnosis on the first visit? And the answer is: By taking a history, such as we have outlined at the beginning of this paper, together with a careful ophthalmic examination, supplemented by the $\mathrm{X}$-ray. This is the exacting path that must be trav-
One must have an X-ray in all doubtful cases. To emphasize this point strongly, we cite three cases in this series, with normal vision and in which the one positive finding was a complaint of severe pain. It was this, and the nature of the work engaged in when injured, both of which were elicited in the history, that prompted us to have an X-ray.

It was soon after Prof. Roentgen published his classical papers that Dr. Campbell first had an opportunity to make use of the X-ray as a method of ocular diagnosis. A man who had re- 
cently been injured with a piece of steel was seen at the office in the early part of the year 1897. His eye was deeply inflamed and the vision reduced. Was there a piece of steel inside of that eye and, if so, in what part of the eye was it located?

At that time Detroit, if not the entire state of Michigan, could boast of but one X-ray machine in its vicinity. It was owned, not by a medical man, but by a Mr. S. M. Keenan, who had his apparatus set up at Eloise, Michigan. It was there that our first X-ray of a metallic foreign body retained inside of the cye was taken. At that time Sweet" had not perfected his method of localization, the accuracy of which, now, in the hands of one who is skilled in that work, is almost uncanny. But by this new procedure it was possible to state positively that the man had a piece of stecl inside of his eye and, in a degree, determine its location. A scleral puncture was made and the steel removed by the use of the early type of Hirschberg hand magnet. This is one of the earliest, if not the first case, in which a metallic foreign body was located within the eye by the X-ray, and successfully removed by the use of the magnet-a few months over a year from the date that Roentgen first announced his discovery of the X-ray. There was a case reported by Dr. Chas. R. Williams, ${ }^{3}$ in Boston, which precedes this case by a few months. They identified the foreign body by the X-ray; but unfortunately it was not magnetizable and so could not be removed by the use of the magnet.

Since this early date we have had excellent service from the X-ray. If we consider our scries from the point of the X-ray, we see that, out of 1,051 cases, 117 or about 11.1 per cent required an X-ray before a positive diagnosis could be made. In no case have we failed to diagnose steel inside of the eye. Of the 117 cases X-rayed, 17 per cent were positive for steel inside of the eye, and all of these were diagrnosed on the date of their first visit in the office.
It is a point of some practical importance to emphasize that all the positive X-rays occurred in the steel group; also of the 438 cases injured while working on the emery wheel, only two required an X-ray and both of those were negative. With this in mind, we can more intelligently advise concerning the need for an X-ray.

\section{REMOVAI. OF FOREIGN BODY INSIDE THE} EYE.

Once having diagnosed and localized a foreign body inside the eye, how shall we proceed to remove it? As we read the literature, we find a great difference of opinion on this question. The great majority of such foreign bodies are metals, which are attracted by the magnet. Such being the case, all are agreed that the most expedient means of attack is by use of the magnet.

Shall we use a large or a small magnet? Remove the foreign body thru the anterior chamber, known as the anterior route, or by a scleral puncture, known as the posterior route? These are questions on which oculists are divided. and are questions which will be of little profit to discuss here. Rather, we will point out a few of the more interesting and important features as they have occurred in the magnet work for this series.

We have had but one case where we were not able to remove the steel by the magnet. This was a case where a very small piece of steel was embedded in the ciliary body. Two attempts were made to remove this, first thru a scleral puncture just back of the ciliary body, and second thru a keratome incision in the cornea, both of which failed. After a stay of ten days in the hospital the man had a normal vision, the congestion in the eye had cleared ap, and there was no pain. There were two things one might do in this case. First, one could cut down thru the ciliary body and possibly get the steel. The other plan would be to allow the steel to remain in the muscular coat of the eye and observe it for irritation or siderosis. We chose the latter, and when the case was last scen, 10 
months after the injury, he was having no trouble with the cye.

We have had in this series four cases of steel embedded in the lens. If the steel is completely embedded in the substance of the lens, we have preferred to wait until the cataract is mature and then, by extracting the cataract, remove the steel at one and the same operation. In this series we had one such case with a vision corrected by glasses to $6 / 5$ following the operation, or $2 / 10$ better than normal vision. If one end of the foreign body is sticking in the lens, leaving a portion of the metal in contact with the aqueous, we then proceed to remove the foreign body at once thru a keratome incision in the cornea. In this group there are three cases of steel in the lens which were removed in the above manner. Two developed traumatic cataracts, while

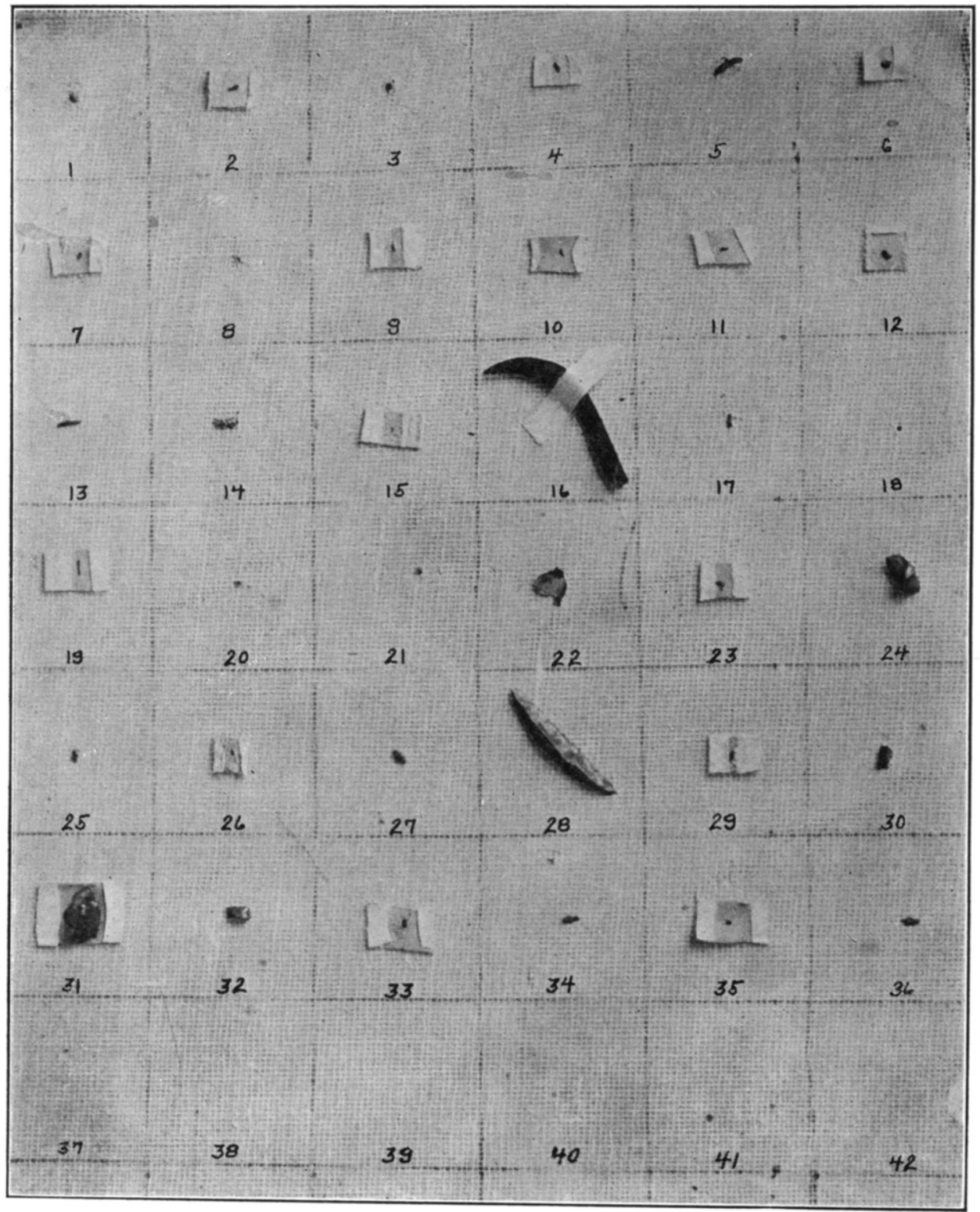

Fig. 3.-Specimens of stcel removed from inside the eye by giant magnet, two-thirds actual size. 
in one case the lens remained clear up to 4 or 5 months following the injury, at the time last seen in the office.

$A$ foreign body located in the anterior or posterior aqueous chambers, we believe, should, in practically all cases, be removed thru the cornea. In cases where the foreign body has passed thru the cornea and the lens with a resulting traumatic cataract, and it is shown by the X-ray to be located in the vitreous or in the coats of the eye, it may be removed by the anterior route, if the foreign body is not so large as to unduly endanger an entanglement of the iris or ciliary body. In most of our cases localized in the vitrcous, we have removed the foreign body thru the sclera. Certainly, if the anterior segment of the eye is intact and the lens is not injured, we would remove the foreign body thru the sclera, and not drag it forward as Haab advises.

We wish to report on 24 cases of steel inside of the eye, four of which did not occur in this series. Eleven of the 24 or about 50 per cent had a vision of $6 / 7$ to .5 , or practically normal, following the operation for removal of the steel. Six of these were operated by the posterior route, four by the anterior route, and in one the steel was removed with the lens at the time of the cataract operation. Fight of these cases have been seen within the last few months at the office, ranging from ten to eighteen months since their operation, and the vision given is on the basis of this last examination. Nine of the 24 following the removal of the steel had the injured eye enucleated, either because of infection or irritation. Two others were advised to have this operation, but refused it, and one because of the extensive scarring on the cornea had a vision of less than $1 / 10$ normal. This compares very favorably with the reports we find in the literature.

The after-treatment of the case is of no little importance, and is more diffcult to carry out than in most surgical cases. A working man who fecls well will find it difficult to understand why he should be in bed and on his back for ten days to two weeks, following the removal of the smallest piece of steel from his eye. But experience has taught us that such precaution is well taken. We believe detachment of the retina is reduced to a minimum by such a procedure.

For the sake of completeness, it cannot be a mistake to say a few words concerning the treatment of the minor injuries. Here three points are worthy of special mention: 1. Relative to the removal of foreign bodies embedded in the outer coats of the eye. By far the greatest number of these will be found on the cornea. 2. In removing any foreign body from the eye, only sterile instruments should be used. 3. The foreign body should be completely removed. Any stain or pigment left at the margins of the wound will keep up the irritation and cause the patient a great deal of discomfort. In our eagerness to remove all the forcign substance, we should disturb the layers of the cornea as little as possible, and all cases should be followed up and observed for infection.

To summarize the points of interest and practical importance:

1. In this series we have two great classes of work responsible for most of our cases, namely, emery grinding and stcel or metal work, the two totaling 81.3 per cent of all the injuries.

2. That by far the greatest number of our major injuries occur in the second group, i. e., those working on metals.

3. Taking the series as a whole, about one case in every thirteen, or 7.9 per cent, were major injuries, while in the cases occurring in the steel group one out of every eight cases, or 12.9 per cent, were major injuries, the percentage being much higher in the steel group.

4. That better than one in ten of all our cases have required an X-ray, before we could be positive that a foreign body was not inside the eye.

5. 117 X-rays were taken, and 100 of these occurred in the steel group.

6. 20 , or 17 per cent of the radiograms taken were positive for foreign 
body inside of the eye, and all these occurred in the steel group.

7. The danger signal is that 71 per cent of our major injuries occurred in the steel group, all our positive $\mathrm{X}$-rays occurred here, and 60 per cent of the eyes lost, or blind, were chargeable to metals. Such figures should cause us to adopt the attitude that all cases injured while working on metals are serious, and to treat them as such.

8. The magnet operation, as such, can be consilered quite successful; we were able to remove the magnetizable foreign body in 95 per cent of the cases.

9. 23.8 per cent of the cases on which we did an iridectomy had a normal vision following the operation.

10. 68.5 per cent of the eyes in which the sight was lost had to be enucleated.

11. Only 17.5 per cent of the cases were seen at our office on the lay they were injured, 43.5 per cent on the second day after their injury, 17.5 per cent on the third day, while better than $1 / 5$ of the cases, or 21.5 per cent waited four days or longer, before they applied at the office for treatment. These last figures, we believe, are very $1 \mathrm{~m}$ portant. Delay in the making of a correct diagnosis, and delay in the instituting of proper treatment are two of the most potent factors responsible for permanent injury to the eye, and are responsible for not a few of the artificial eyes that we sce.

If the results here reported are to be classed as a meritorious showing, certainly the credit is to be shared with those surgeons and general practitioners who early in their cases, have realized the necessity for special examination or treatment. If the number of eyes enucleated or permanently impaired are to be materially lessened, then we must strive to obtain a higher grade of ophthalmic judgment, not only in the oculist but also in the general medical profession. The delay in correct diagnosis and proper treatment must approach a minimum.

\section{REFERENCFS,}

1. Wm. A. Swect. Journal, A. M. A., 1906, p. 359.

2. Wm. A. Sweet. The Ophthalmoscope. Jan., 1906.

3. Charles R. Williams. Boston Med. \& Surg. Journal. Ang., 13, 1896.

4. Haab. Trans. Sec. of Ophth. A. M. A. 1902, p. 85. 